\title{
EVALUANDO LA FRONTERA PACAJES-CARANGAS PARA EL PERÍODO INTERMEDIO TARDÍO (1.100-1.450 D.C.) EN EL ALTIPLANO BOLIVIANO CENTRAL A PARTIR DE ANÁLISIS DE PASTAS CERÁMICAS*
}

\author{
EVALUATING THE PACAJES-CARANGAS BOUNDARY FOR THE BOLIVIAN \\ CENTRAL ALTIPLANO IN THE LATE INTERMEDIATE PERIOD \\ (AD 1,100-1,450), BASED ON CERAMIC PASTE ANALYSIS
}

Juan Villanueva Criales ${ }^{1,2}$

\begin{abstract}
Este trabajo evalúa la frontera entre las entidades Pacajes y Carangas del altiplano boliviano central durante el Período Intermedio Tardío (a.p. 1.100-1.450 d.C.), mediante la caracterización petrográfica y DRX de pastas cerámicas procedentes de sitios de torres funerarias. Nuestros resultados sugieren un énfasis en la distinción local y fluidez a través de la postulada frontera durante el Intermedio Tardío, situación distinta a la descrita por la etnohistoria para momentos posteriores.

Palabras claves: período Intermedio Tardío, Andes sur-centrales, cerámica, fronteras, arqueometría.
\end{abstract}

This paper evaluates the Pacajes-Carangas boundary in the Bolivian central altiplano during the Late Intermediate Period (ca. AD 1,100-1,450), based on petrographic and RXD characterization of ceramic pastes from burial tower or chullperio sites. Our results suggest an emphasis toward local distinction and cross-boundary fluency, differing from the situation described for later moments by ethno-historical accounts.

Key words: Late Intermediate Period, south-central Andes, pottery, boundaries, archaeometry.

El altiplano boliviano central es la porción de la meseta altiplánica ubicada entre la cuenca del lago Titicaca y la región intersalar (Figura 1). El Período Intermedio Tardío (ap. 1.100-1.450 d.C.) en esta región es un lapso, escasamente estudiado, entre el colapso del Estado Tiwanaku y la conquista Inka, y actualmente suele considerarse que estas poblaciones tienen su origen en la cuenca del Titicaca (Janusek 2003; Pärssinen 2005) o en procesos locales del altiplano central (Michel 2000). En ese contexto se ha asumido tácitamente la existencia de una frontera étnica entre los "señoríos" de Pacajes y Carangas descrita por la etnohistoria, siendo que los indicadores arqueológicos, especialmente de forma y decoración cerámica, sugieren una homogeneidad regional (Villanueva 2013). Este trabajo evalúa la mencionada frontera regional durante el Intermedio Tardío, a partir de la caracterización de las pastas que componen la cerámica empleada en ciertas ceremonias grupales. Para ello, se establecen los antecedentes de estudio, se describen los procedimientos empleados, y se describen y discuten brevemente los resultados alcanzados.

\section{Antecedentes y Problemática}

Una característica del altiplano central durante el PIT es la presencia de chullperios, sitios funerario-ceremoniales con presencia de torres funerarias o chullpares. Referencias etnohistóricas y etnográficas (Gil 2001; Isbell 1997) describen el ritual comunitario que tenía lugar en el espacio de los chullpares, resaltando su rol en la reproducción identitaria mediante el consumo de comida y bebida.

Como resultado de estas actividades, los chullperíos presentan cerámica fragmentada superficial, reportada tempranamente por la arqueología boliviana (Bennett 1936; Ryden 1947). Con el nombre de Pacajes (Portugal 1988), este material fue reportado en las décadas de 1990 y 2000 en la cuenca del Titicaca, fechado en el Intermedio Tardío (Albarracín 1996; Janusek 2003).

\footnotetext{
* Artículo seleccionado del conjunto de ponencias presentadas en el III Congreso Latinoamericano de Arqueometría, realizado en Arica, Chile, el año 2011. Este manuscrito fue evaluado por investigadores externos y editado por Marcela Sepúlveda y Verónica Silva, en su calidad de editoras invitadas de la Revista.

1 Programa de Doctorado en Antropología UCN-UTA, Universidad de Tarapacá, Campus Azapa, Arica, Chile.

2 Casilla 08028, Correo Central, La Paz, Bolivia. juan710@ gmail.com
} 


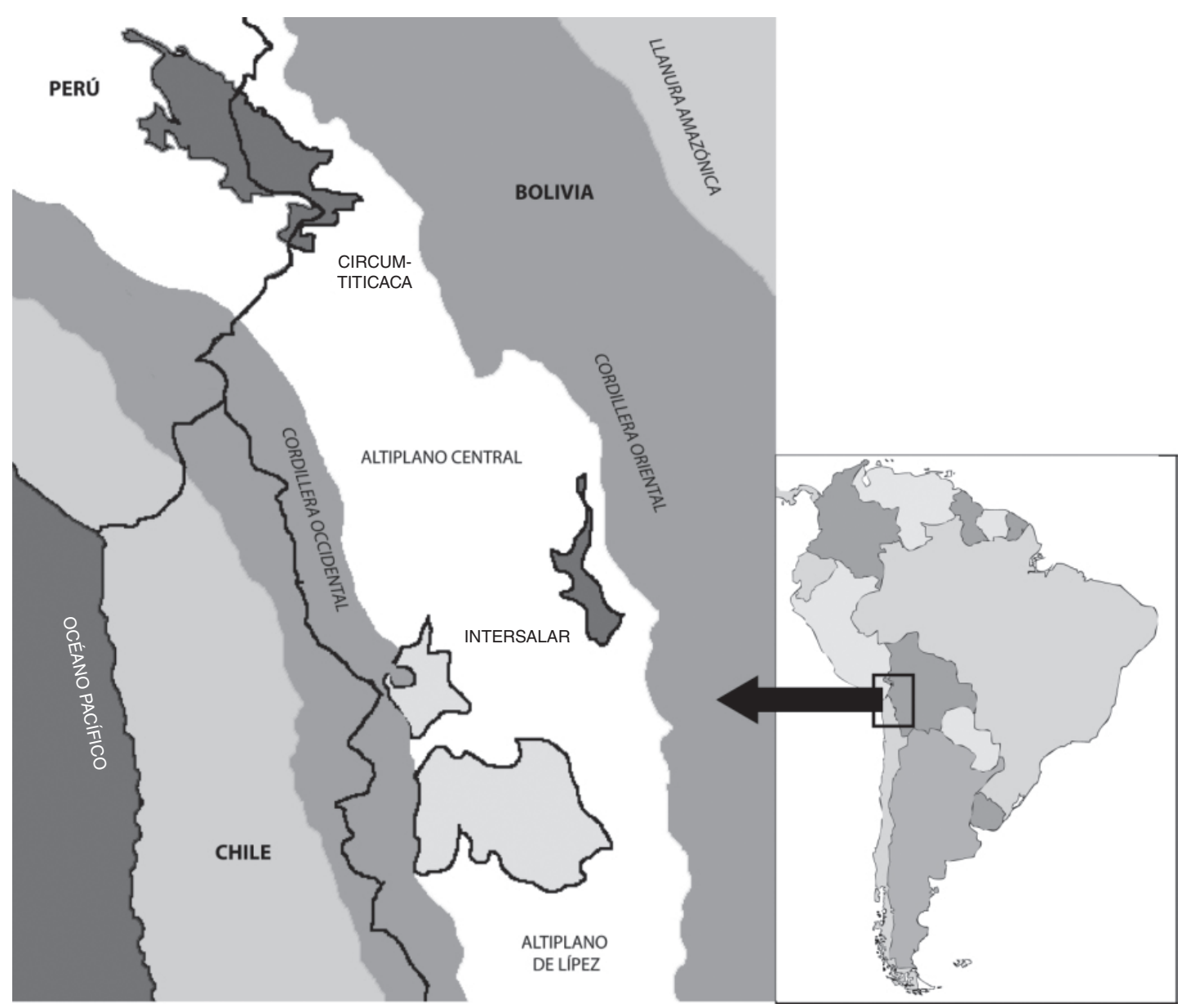

Figura 1. Ubicación del altiplano boliviano central. Location of central Bolivian altiplano.

La cerámica Pacajes es luego documentada en el altiplano central (Pärssinen 2005), región cuya arqueología tiene fuerte influencia etnohistórica. Los documentos coloniales describen un espacio dividido por la frontera del río Mauri-Desaguadero entre los "señoríos" de Pacajes y Carangas (BouysseCassagne 1987; Saignes 1986). Esta frontera étnica ha sido extrapolada al estudio del Intermedio Tardío en la región, sugiriendo, en el caso de Carangas, la presencia de organizaciones locales de tipo "señorío" desde tiempos formativos (Michel 2000). El uso del modelo etnohistórico ha causado que las investigaciones realizadas en Pacajes (Kesseli y Pärssinen 2005; Pärssinen 2005; Sagárnaga 2008; Villanueva y Patiño 2008) y Carangas (Díaz 2003; Gisbert 2001; Michel 2000) se ignoren mutuamente, asumiendo una frontera arqueológicamente invisible: una revisión de la bibliografía delata ausencia de diferencias materiales claras entre Pacajes y Carangas, en términos de patrones de asentamiento y arquitectura, y de forma y decoración cerámica (Figura 2).

En este trabajo estudiamos las pastas cerámicas presentes en los chullperíos, como resultado de ceremonias comensales comunitarias. Entendemos que la utilización de determinado material cerámico en estos eventos implicó el acceso a ciertas fuentes de materiales y/o la interacción social con determinados grupos de alfareros, posiblemente afines y pertenecientes al mismo colectivo social mayor. La existencia de una frontera rígida entre dos grupos sociales cerrados implicaría una distribución de materiales diferentes a ambos lados del río Mauri-Desaguadero. 

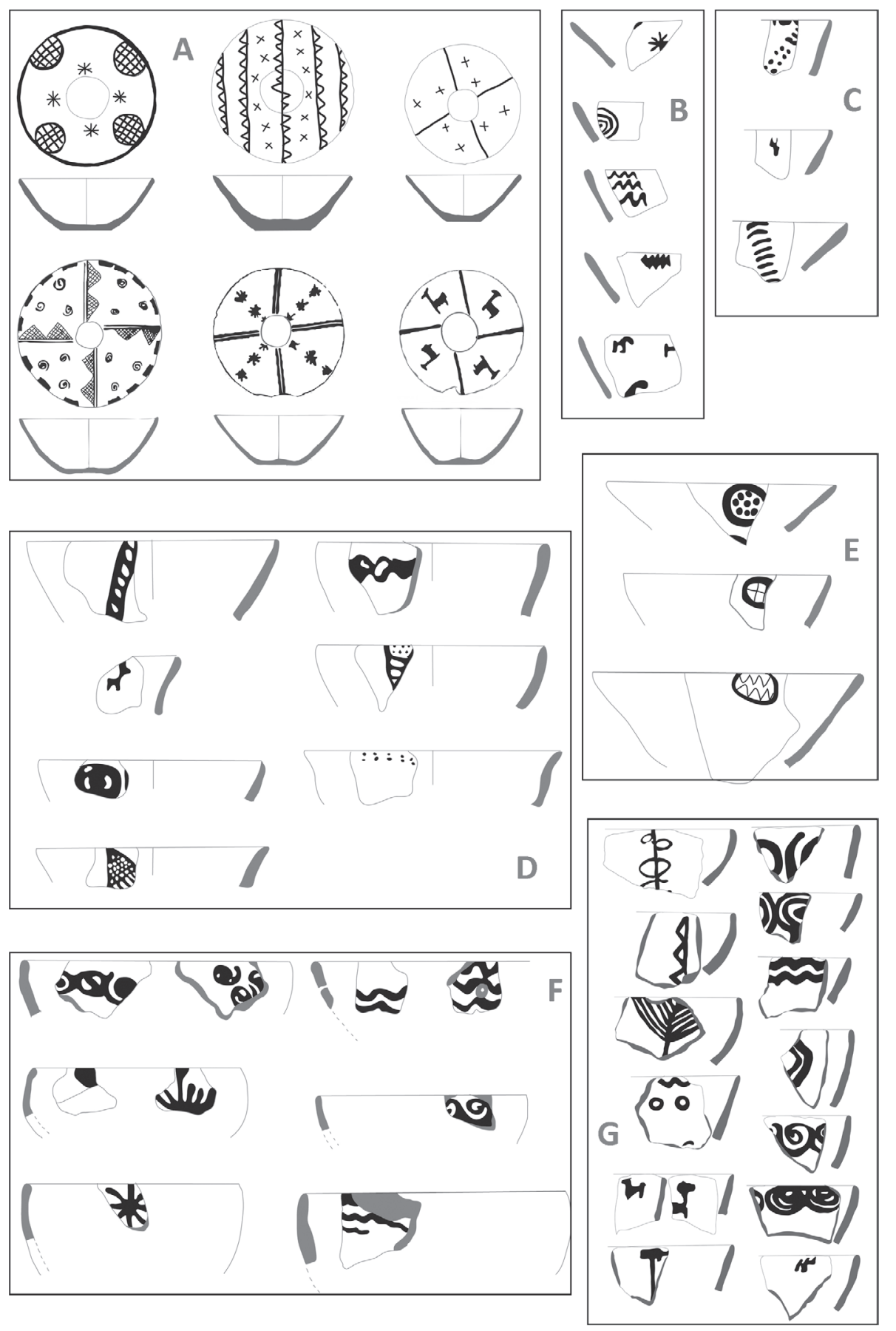

A: Pacajes (Villanueva y Patiño 2008) E: Uma Pacajes (Janusek 2003)

B: Pacajes (Pärssinen 2005)

F: Carangas (Díaz 2003)

C: Pacajes (Albarracín 1996)

G: Carangas (Michel 2000)

D: Urco Pacajes (Janusek 2003)

Figura 2. Comparación entre decoración cerámica de Pacajes y Carangas.

Comparison between ceramic decoration from Pacajes and Carangas. 


\section{Muestra y Métodos}

Se ha trabajado con material de tres chullperíos ubicados transversalmente al río Desaguadero. Estos sitios son Condoramaya en Pacajes, Callapa Chica a orillas del mencionado río, y Choquemarca en Carangas (Tabla 1; Figura 3). Los sitios fueron objeto de recolecciones superficiales sistemáticas de cerámica diagnóstica. El material que constituyó la muestra para el análisis macro fue de 200 tiestos, 100 de Condoramaya, 44 de Callapa Chica y 56 de Choquemarca.

Los tiestos se analizaron macroscópicamente, enfatizando las características tecnológicas, y logrando una definición cualitativa de 10 pastas cerámicas mediante criterios de textura, dureza, porosidad, e inclusiones. A partir de los tipos de pasta definidos se seleccionó una muestra de 16 fragmentos para cortes de sección delgada y análisis petrográfico. Este análisis permitió caracterizar las inclusiones en términos de distribución, forma y tamaño, y ponderar su cantidad.

Las pastas fueron reagrupadas por estos resultados, y una muestra de cada grupo fue pulverizada separando la pasta de otros componentes, y analizada mediante DRX, para caracterizar y cuantificar las fases minerales del material y validar la clasificación petrográfica. Finalmente se reanalizó la base de datos general para evaluar las relaciones estadísticas entre la nueva clasificación de pastas y otras variables.

\section{Resultados}

El análisis petrográfico mostró pastas con porcentajes bajos de inclusiones y distribuciones equilibradas a muy buenas. Las inclusiones oscilan entre muy finas y medias, sugiriendo que se encontrarían naturalmente añadidas a las arcillas.

Las pastas tienen un repertorio similar de inclusiones: cuarzo, biotita y feldespatos, además de materiales volcánicos -andesitas, muscovitas y vulcanitas- y vidrio volcánico. Esto sugiere que las arcillas pertenecen a un mismo entorno geológico, aunque nuestro conocimiento de la geología regional aún no permite sugerir hipótesis de procedencia. Aparte de estas similitudes, se detectó variabilidad al interior de la muestra en la proporción relativa de las inclusiones, y en algunos casos en ciertos aspectos cualitativos, permitiendo definir cinco grupos de pasta (Tabla 2; Figura 4).

Los resultados DRX no consideran la proporción de material vítreo que por no poseer estructura cristalina no pudo ser ponderado. El análisis ha revelado la existencia de seis fases mayoritarias para todas las muestras: calcita, cuarzo, anortita, muscovita, feldespato y hematita. Esto sugiere una vez más pertenencia al mismo entorno geológico. Sin embargo, se han observado diferencias significativas en la frecuencia de estos minerales, evaluadas mediante T de Student (Tabla 3). Esto valida la clasificación petrográfica realizada para su uso en la base de datos general.

La clasificación petrográfica de pastas cerámicas se relaciona significativamente con las variables de sitio de procedencia y forma cerámica, siendo además la única característica técnica que exhibe estas relaciones. Los tres sitios exhiben presencia de las cinco pastas, existiendo tendencias cuantitativas de distribución (Figura 5). Revisando las desviaciones estándar se ve que la pasta $\mathrm{C}$ es la más homogéneamente distribuida, mientras que las restantes varían más y se asocian a Choquemarca (pasta B), Callapa Chica (D), y Condormaya (A y E). Además, las pastas B y E tienden a disminuir gradualmente en términos espaciales, la pasta B en dirección sur-norte, y la $\mathrm{E}$ de modo inverso.

Las cinco pastas han sido empleadas en la manufactura de las tres formas principales -cuencos, cántaros y jarras de un asa-, sin existir una especialización funcional de las pastas (Figura 6). La revisión de desviaciones estándar sugiere que la

Tabla 1. Características de los sitios estudiados.

Characteristics of the sites studied.

\begin{tabular}{lcccccccc}
\hline & $\begin{array}{c}\text { Coordenadas UTM } \\
(\text { WGS84) }\end{array}$ & $\begin{array}{c}\text { Altitud } \\
(\mathrm{msm})\end{array}$ & $\begin{array}{c}\text { Superficie } \\
\text { (hectárea) }\end{array}$ & Localización & $\begin{array}{c}\mathrm{N}^{\mathrm{o}} \text { de } \\
\text { sectores }\end{array}$ & $\begin{array}{c}\mathrm{N}^{\mathrm{o}} \text { de } \\
\text { torres }\end{array}$ & Orientación & Referencias \\
\hline Condoramaya & $19 \mathrm{~K} 590452-8080987$ & 3.970 & 2,5 & coluvio superior & 5 & 21 & E & Sagárnaga 2008 \\
Callapa Chica & 19K 570188-8064489 & 3.900 & 0,9 & planicie aluvial & 2 & 15 & NE & Michel 2000 \\
Choquemarca & 19K 526364-8000934 & 4.060 & 2,5 & coluvio inferior & 5 & 23 & E \\
\hline
\end{tabular}




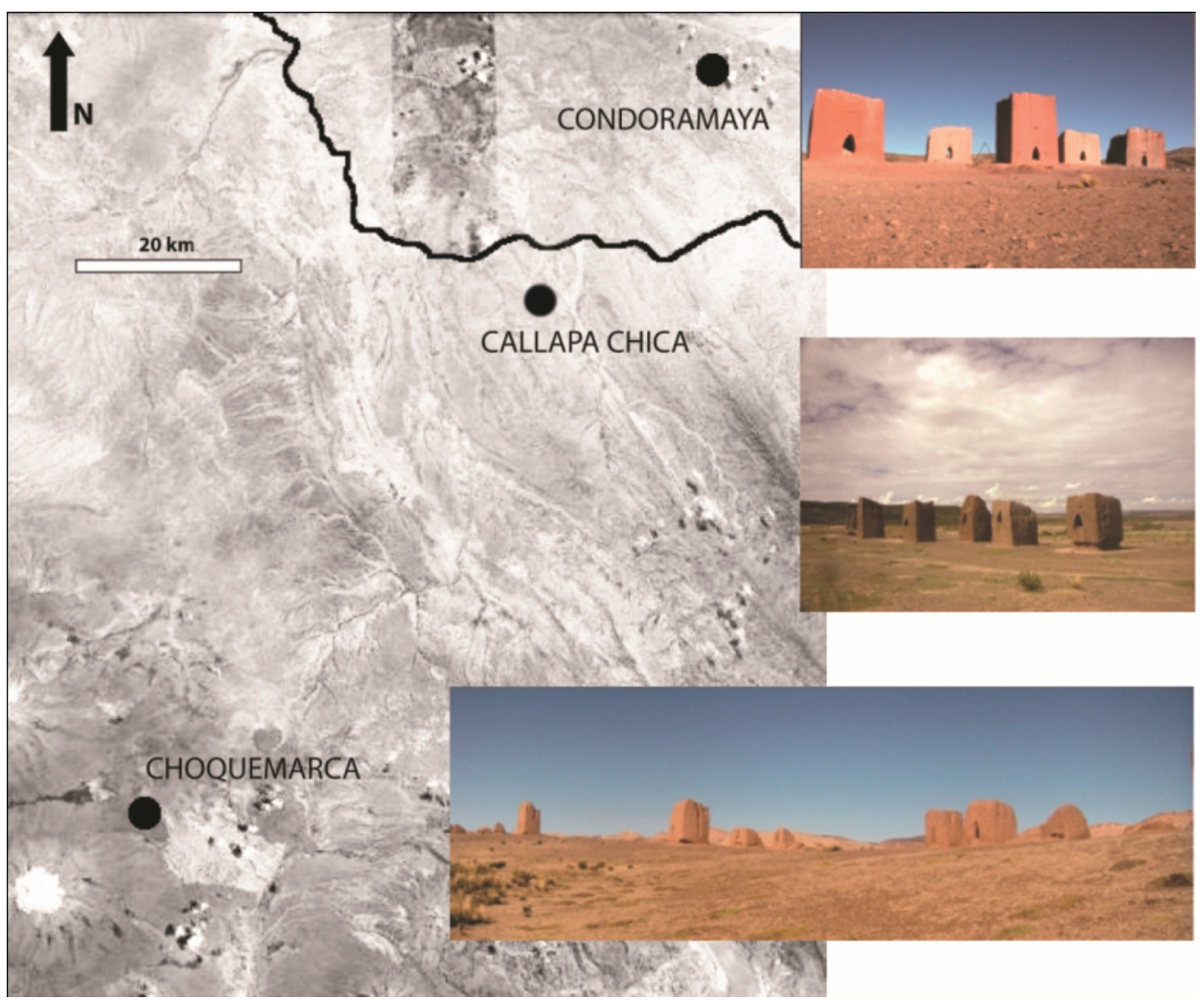

Figura 3. Ubicación de los sitios estudiados. Location of sites studied.

Tabla 2. Características de los cinco grupos de pasta definidos. Characteristics of the five paste groups defined.

Grupo A Presencia de vidrio volcánico en diferentes proporciones. Escasa presencia de otros materiales volcánicos.

Grupo B Ausencia total de vidrio volcánico. Presencia de otros materiales volcánicos en distintas proporciones.

Grupo C Pastas muy seleccionadas, con muy escasa cantidad tanto de vidrio volcánico como de otros materiales volcánicos. Cuarzos redondeados.

Grupo D Inclusiones de mayor granulometría (gruesa a media). Las muestras presentan abundante material volcánico.

Grupo E Se caracteriza por la presencia de cavidades rellenas de carbonatos, que corresponden a microfósiles de esponjas.

Tabla 3. Proporciones de fases minerales en muestras según DRX. XRD mineral phase proportions in samples.

\begin{tabular}{|c|c|c|c|c|c|c|c|c|}
\hline & Grupo A & Grupo B & Grupo C & Grupo D & Grupo E & Promedio & $\begin{array}{l}\text { Desviación } \\
\text { Estandar }\end{array}$ & $\begin{array}{c}\mathrm{P} \\
\text { (T de Student) }\end{array}$ \\
\hline Calcita & 2 & 1,6 & 1,7 & 1,3 & 1,5 & 1,62 & 0,26 & 0,0002 \\
\hline Cuarzo & 27,9 & 28,1 & 29,9 & 20,5 & 38,6 & 29 & 6,47 & 0,0006 \\
\hline Anortita & 30,1 & 24,8 & 21,9 & 18,7 & 27,5 & 24,6 & 4,49 & 0,0003 \\
\hline Muscovita & 26,7 & 23,9 & 21,7 & 26,2 & 16,7 & 23,04 & 4,07 & 0,0002 \\
\hline Feldespato & 9,1 & 20,8 & 19,6 & 29,8 & 11,8 & 18,22 & 8,17 & 0,0076 \\
\hline Hematita & 4,2 & 0,8 & 5,2 & 3,5 & 3,9 & 3,52 & 1,65 & 0,0088 \\
\hline
\end{tabular}



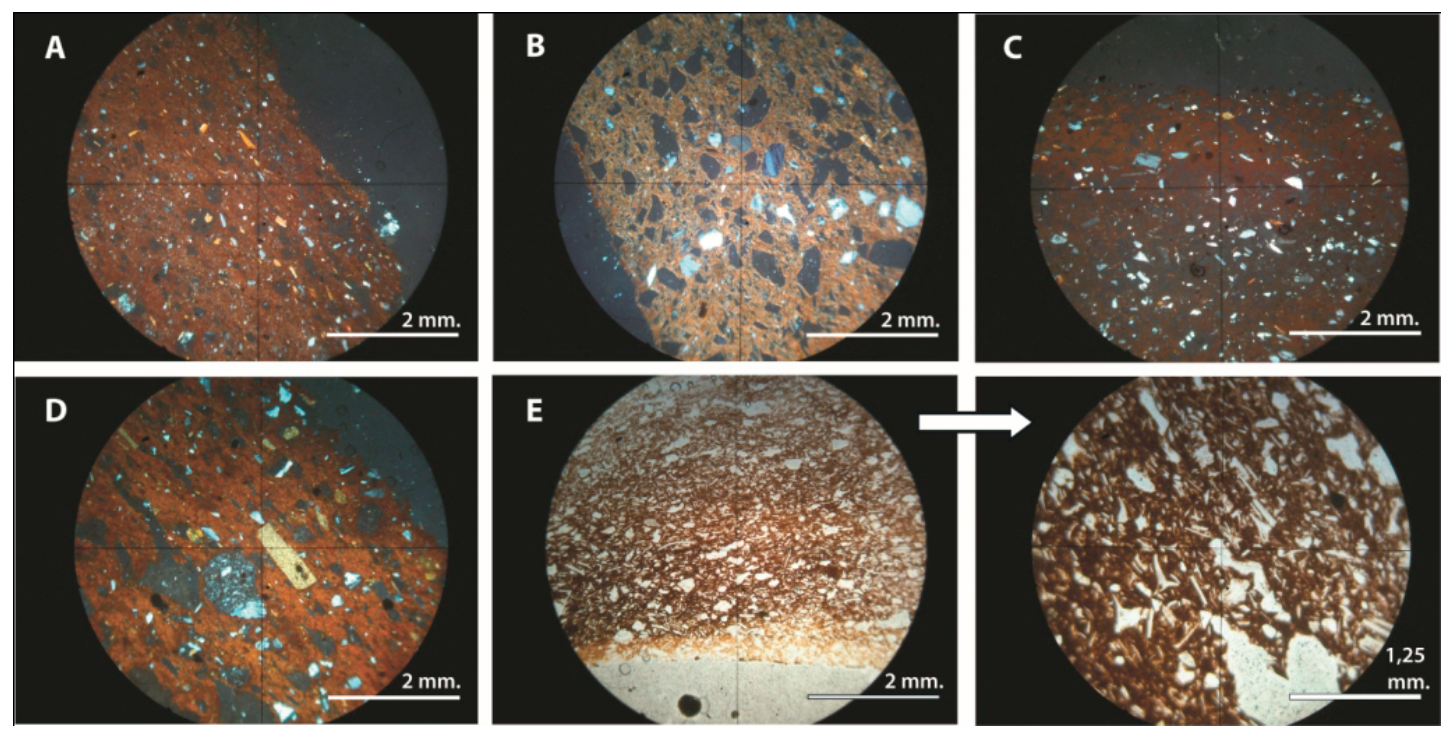

Figura 4. Microfotografías de los cinco grupos de pasta definidos. Micro-photographs of the five paste groups defined.

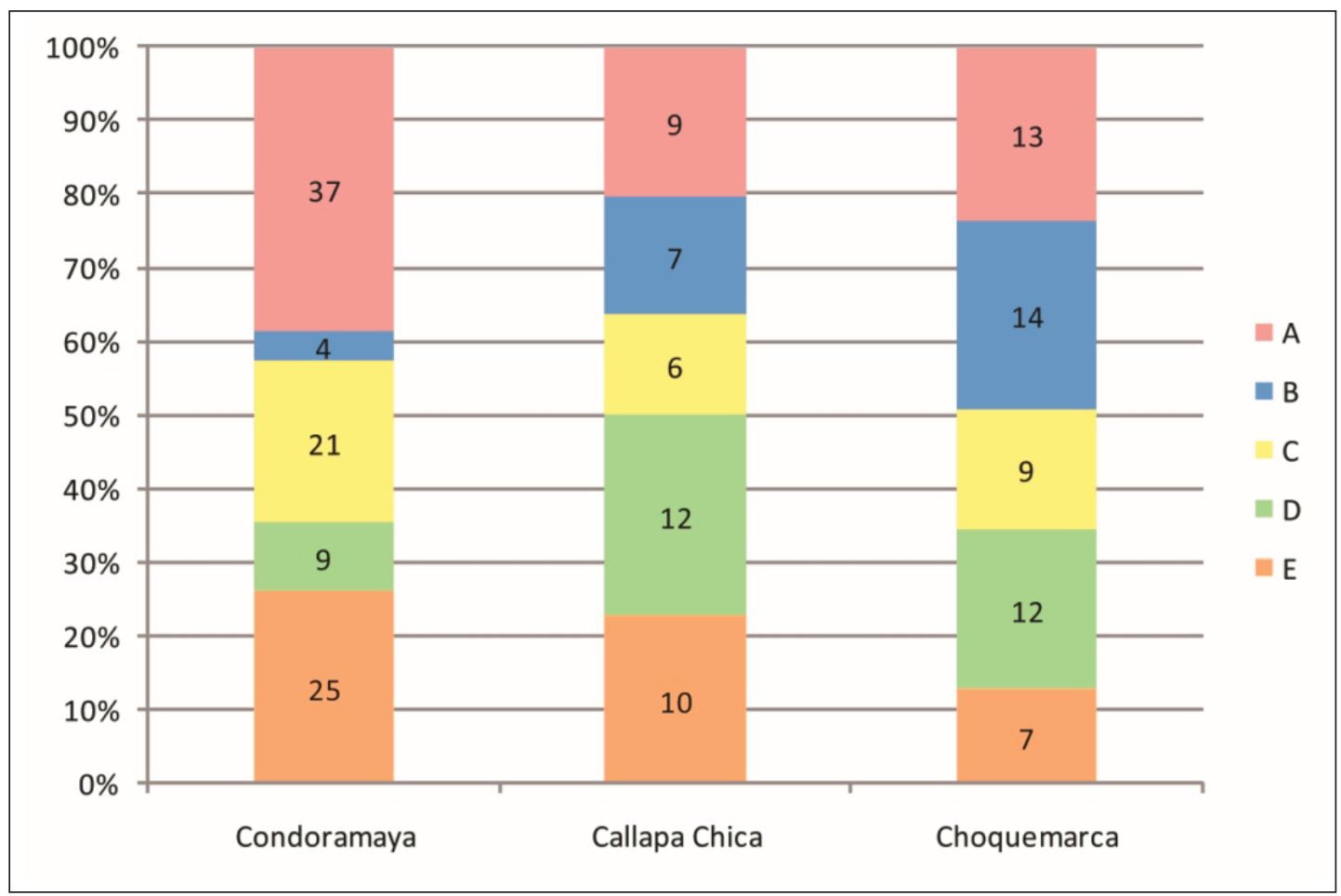

Figura 5. Distribución de pastas cerámicas según sitio de procedencia. Ceramic pastes distribution according to site of origin. 


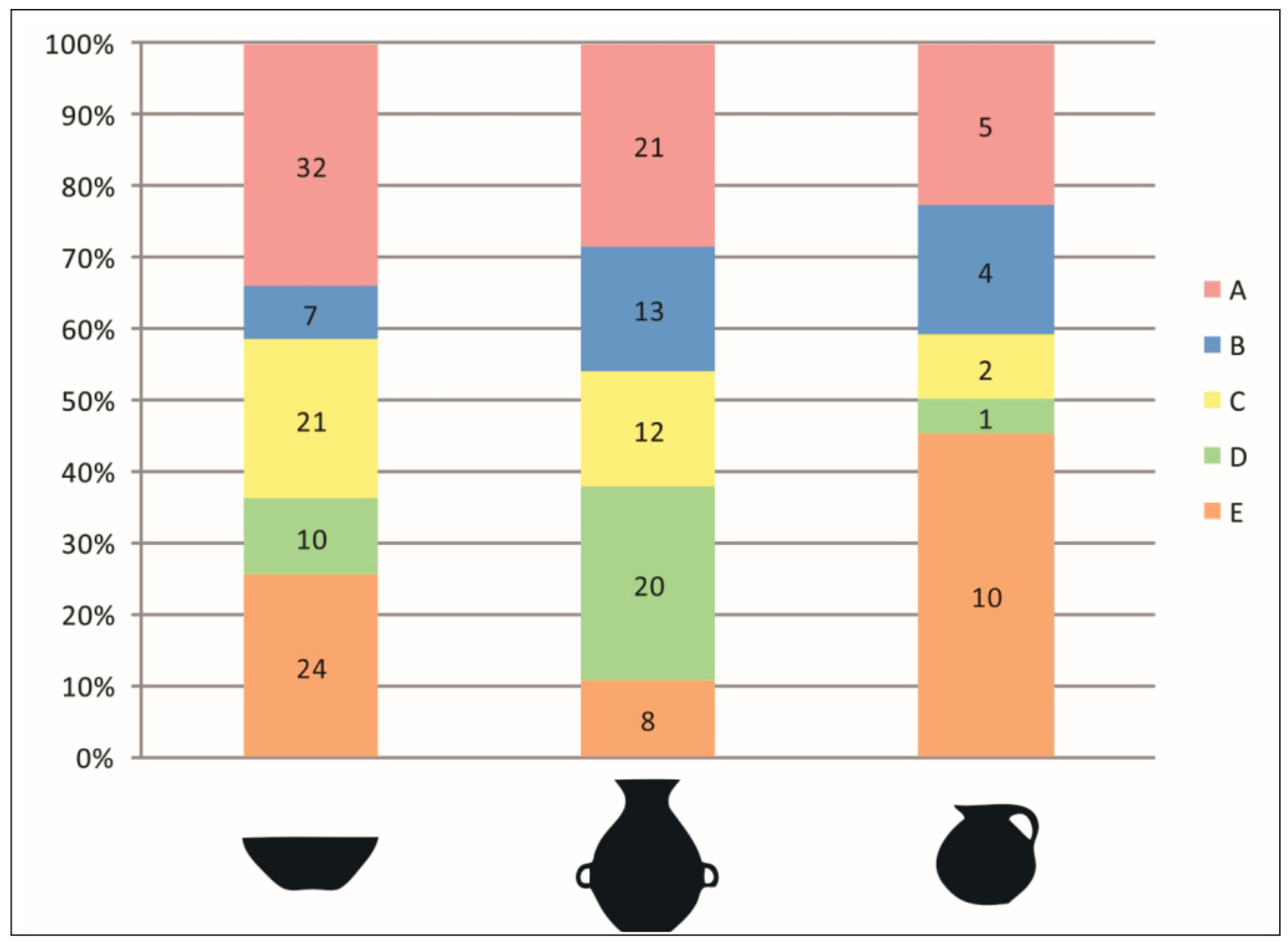

Figura 6. Distribución de pastas cerámicas según forma cerámica. Ceramic pastes distribution according to ceramic shape.

Tabla 4. Tendencias de distribución de pastas y formas cerámicas según sitios. Ceramic paste and shape distribution tendencies, according to sites.

\begin{tabular}{|c|c|c|c|c|c|c|c|c|c|}
\hline & \multicolumn{3}{|c|}{ Condoramaya } & \multicolumn{3}{|c|}{ Callapa Chica } & \multicolumn{3}{|c|}{ Choquemarca } \\
\hline & cuenco & cántaro & jarra & cuenco & cántaro & jarra & cuenco & cántaro & jarra \\
\hline Pasta A & ++ & ++ & - & ++ & - & + & ++ & + & + \\
\hline Pasta B & & - & & + & + & & + & + & ++ \\
\hline Pasta C & + & - & - & - & + & + & + & - & \\
\hline Pasta D & - & - & & - & ++ & & ++ & ++ & - \\
\hline Pasta E & + & + & + & ++ & - & ++ & + & & ++ \\
\hline
\end{tabular}

pasta E está repartida de modo menos homogéneo, vinculada a las jarras. Las tendencias de cada sitio por usar combinaciones de pasta y forma son también variadas (Tabla 4). Choquemarca emplea pastas B y E en las jarras, y cuencos y cántaros en pastas D y A. En Callapa Chica, la preferencia es por pasta $\mathrm{D}$ en los cántaros, E en jarras y A en cuencos. En Condoramaya, la tendencia muestra pasta A para cántaros, $\mathrm{C}$ para cuencos y $\mathrm{E}$ para jarras.

\section{Conclusiones}

La caracterización de materias primas ha mostrado la existencia de variabilidad técnica al interior de la cerámica del Intermedio Tardío en el altiplano central, subyacente a la homogeneidad morfológico-decorativa regional. Esta variabilidad parece provenir de la misma región altiplánica, sin influjos de regiones vecinas. La variabilidad 
en pastas, probablemente originada en las fuentes mismas de arcilla, señala al aprovisionamiento de material como un acto técnico significativo.

El análisis realizado señala que los grupos locales exhiben tendencias peculiares por el uso de ciertas pastas, formas y combinaciones de forma y pasta, al interior de un abanico de posibilidades disponibles a ambos lados de la supuesta frontera. Por tanto, las prácticas de obtención y uso de cerámica en los sitios estudiados sugieren un énfasis en la diferenciación local y no la pertenencia a un colectivo mayor basado en una separación rígida, entre Pacajes y Carangas.

Los patrones sugieren que la frontera del MauriDesaguadero no existió en tiempos del Intermedio Tardío, o bien fue una línea permeable y fluida que los análisis arqueológicos, incluido el presente, no logran detectar. Cabe considerar que, en términos teóricos, una frontera no es necesariamente una barrera, sino un espacio potencial para la interacción humana (Douglas Taylor 2007). En todo caso, este panorama de particularidades locales y fluidez regional es distinto a aquel sugerido desde la etnohistoria para momentos posteriores.

Agradecimientos: Este artículo sintetiza parte de la tesis de Magíster en Antropología desarrollada para el Programa de la UTA-UCN, en Arica. Agradezco a Marcela Sepúlveda, profesora guía de la tesis, por la orientación y la inserción en el proyecto MECESUP 0801. A Ramiro Matos (UMSA, La Paz) por la preparación de secciones delgadas, a Guillermo de la Fuente (UNCa, Catamarca) por el análisis petrográfico, y a Alberto Riveros y Víctor Galván (UNC, Córdoba) por el análisis DRX. A Jédu Sagárnaga por facilitar el acceso al material de los sitios estudiados, a Emily Stovel y Mauricio Uribe por sus críticas y comentarios, y a los dos revisores anónimos del texto. Durante la redacción, el autor contó con una beca MECESUP2. Se agradece finalmente el apoyo del Convenio de Desempeño HCSA Universidad de Tarapacá-MINEDUC.

\section{Referencias Citadas}

Albarracín, J. 1996. Tiwanaku: Arqueología Regional y Dinámica Segmentaria. Plural, La Paz.

Bennett, W. 1936. Excavations in Bolivia. Anthropological Papers of the American Museum of Natural History, Nueva York.

Bouysse-Cassagne, T. 1987. La Identidad Aymara: Aproximación Histórica. HISBOL/IFEA, La Paz.

Díaz, C. 2003. Pumiri: una Aproximación al Criterio de Poder y Expansión Inca. Tesis de Licenciatura, Carrera de Arqueología, UMSA, La Paz.

Douglas Taylor, L. 2007. El concepto histórico de la frontera. Antropología de las Fronteras. Alteridad, Historia e Identidad más allá de la Línea, editado por M. Olmos, pp. 231-261. El Colegio de la Frontera Norte, México.

Gil García, F. 2001. Secuencia y consecuencia del fenómeno chullpario. En torno al proceso de semantización de las torres chullpa. Anales del Museo de América 9:165-199.

Gisbert, T. 2001. El Paraíso de los Pájaros Parlantes. La Imagen del Otro en la Cultura Andina. Plural, La Paz.

Isbell, W. 1997. Mummies and Mortuary Monuments. A Postprocesual Prehistory of Central Andean social Organization. University of Texas Press, Austin.

Janusek, J. 2003. Vessels, time, and society: Toward a ceramic chronology in the Tiwanaku heartland. En Tiwanaku and its
Hinterland 2, editado por A. Kolata, pp. 30-91. Smithsonian Institution Press, Washington D.C.

Kesseli, R. y M. Pärssinen 2005. Identidad étnica y muerte: torres funerarias (chullpas) como símbolos de poder étnico en el altiplano boliviano de Pakasa (1250-1600 d.C.). Bulletin de l'Institut Français d'Études Andines 34:379-410.

Michel, M. 2000. El Señorío Prehispánico de los Caranga. Tesis de Diplomado Superior en Derecho de los Pueblos Indígenas. Universidad de la Cordillera, La Paz.

Pärssinen, M. 2005. Caquiaviri y la Provincia Pacasa. CIMA, La Paz.

Portugal Ortiz, M. 1988. Informe de la prospección a Pacajes (Etapa 1). Arqueología Boliviana 3:109-117.

Rydén, S. 1947. Archaeological Researches in the Highlands of Bolivia. Eanders Boktryckeri Akiebolag, Götteborg.

Sagárnaga, J. 2008. Allí donde yace el Cóndor: generalidades en torno a la localidad arqueológica de Wayllani/Kuntur Amaya. Chachapuma 3:5-22.

Saignes, T. 1986. En Busca del Poblamiento étnico de los Andes Bolivianos (s. XV y XVII). MUSEF, La Paz.

Villanueva, J. 2013. Materiales Cerámicos y la Construcción Arqueológica de Pacajes y Carangas. Una Evaluación Arqueométrica de la Frontera del Mauri-Desagüadero para el Período Intermedio Tardío en el Altiplano Boliviano Central. UTA/UCN/MECESUP2. Arica. 